\title{
Platinum-Catalyzed Intermolecular Hydroamination of Vinyl Arenes with Carboxamides
}

Hua Qian and Ross A. Widenhoefer*

P. M. Gross Chemical Laboratory

Duke University

Durham, North Carolina 27708-0346

\section{Supporting Information}

Experimental procedures and spectroscopic and analytical data for new compounds and hydroamination products (20 pages). 
General Methods. All reactions were performed in sealed glass tubes under a nitrogen atmosphere. NMR spectra were obtained on a Varian spectrometer operating at 400 $\mathrm{MHz}$ for ${ }^{1} \mathrm{H}$ and $100 \mathrm{MHz}$ for ${ }^{13} \mathrm{C}$ in $\mathrm{CDCl}_{3}$ unless noted otherwise. Gas chromatography was performed on a Hewlett-Packard 5890 gas chromatography equipped with a $25 \mathrm{~m}$ polydimethylsiloxane capillary column. Flash column chromatography was performed employing 200-400 mesh silica gel (EM). Thin layer chromatography (TLC) was performed on silica gel $60 \mathrm{~F}_{254}$ eluting with a 2:1 mixture of hexanes and ethyl acetate unless noted otherwise. IR spectra were obtained on a Bomen MB-100 FT-IR spectrometer. Elemental analyses were performed by Complete Analysis Laboratories (Parsippany, NJ). 1,4-Dioxane (Acros, anhydrous), $\left[\mathrm{PtCl}_{2}\left(\mathrm{H}_{2} \mathrm{C}=\mathrm{CH}_{2}\right)\right]_{2}$ (1) (Strem), phosphines (Strem), 2-oxazolidone, $p$ toluenesulfonamide, benzo[1,3]dioxole-5-carboxamide, and commercially available carboxamides and vinyl arenes (Acros or Aldrich) were used as received. Mesitylene was distilled over $\mathrm{CaH}_{2}$ under nitrogen prior to use.

\section{Substrates}

Methyl terephthalamate (Table 2 , entry 6). ${ }^{1} \quad$ A solution of methyl 4chlorocarbonylbenzoate $(1.0 \mathrm{~g}, 5.0 \mathrm{mmol})$ in THF $(15 \mathrm{~mL})$ was added slowly to concentrated aqueous ammonium hydroxide $(16 \mathrm{~N}, 30 \mathrm{~mL})$ at $0{ }^{\circ} \mathrm{C}$ and the resulting suspension was stirred for $1 \mathrm{~h}$. The resulting precipitate was collected by filtration and recrystallized from methanol to give methyl terephthalamate as colorless needles $(0.43 \mathrm{~g}, 48 \%) . \mathrm{mp} 210-212^{\circ} \mathrm{C}$. ${ }^{1} \mathrm{H}$ NMR $\left(\mathrm{DMF}-d_{7}\right): \delta 8.32(\mathrm{br} \mathrm{s}, 1 \mathrm{H}), 8.14(\mathrm{~d}, J=8.8 \mathrm{~Hz}, 2 \mathrm{H}), 8.08(\mathrm{~d}, J=8.8 \mathrm{~Hz}, 2 \mathrm{H})$, 7.64 (br s, $1 \mathrm{H}), 3.94(\mathrm{~s}, 3 \mathrm{H}) .{ }^{13} \mathrm{C}\left\{{ }^{1} \mathrm{H}\right\} \mathrm{NMR}\left(\mathrm{DMF}-d_{7}\right): \delta 168.5,167.0,139.8,133.3$, $130.3,128.9,53.0$. 
4-(tert-Butyldimethylsiloxy)benzamide (Table 2, entry 13). A solution of 4hydroxybenzamide (1.5 g, $10.9 \mathrm{mmol})$, TBDMSCl $(2.0 \mathrm{~g}, 13.3 \mathrm{mmol})$, and imidazole $(1.5 \mathrm{~g}$, $22.0 \mathrm{mmol})$ in DMF (25 mL) was stirred at room temperature for 2 days. The reaction mixture was quenched with water and extracted with chloroform. The combined organic extracts were washed (water), dried $\left(\mathrm{MgSO}_{4}\right)$, and concentrated under vacuum. Chromatography of the residue $\left(\mathrm{SiO}_{2} ;\right.$ hexanes-ethyl acetate $\left.=2: 1\right)$ gave 4 - $($ tertbutyldimethylsiloxy)benzamide $(1.72 \mathrm{~g}, 63 \%)$ as a white solid. $\mathrm{mp} 143-144{ }^{\circ} \mathrm{C} . \mathrm{TLC}: \mathrm{R}_{f}=$

0.11. ${ }^{1} \mathrm{H}$ NMR: $\delta 7.72(\mathrm{~d}, J=8.8 \mathrm{~Hz}, 2 \mathrm{H}), 6.87(\mathrm{~d}, J=8.8 \mathrm{~Hz}, 2 \mathrm{H}), 5.95$ (br s, $\left.2 \mathrm{H}\right), 0.98$ (s, $9 \mathrm{H}), 0.22(\mathrm{~s}, 6 \mathrm{H}) .{ }^{13} \mathrm{C}\left\{{ }^{1} \mathrm{H}\right\}$ NMR: $\delta \quad 169.4,159.5,129.5,126.6,120.4,25.9,18.6,-4.1$. IR (neat, $\mathrm{cm}^{-1}$ ): 3405 (s), 1653 (s). Anal. Calcd (found) for $\mathrm{C}_{13} \mathrm{H}_{21} \mathrm{NO}_{2} \mathrm{Si}: \mathrm{C}, 62.11$ (61.97); $\mathrm{H}, 8.42(8.58) ; \mathrm{N}, 5.57(5.71)$.

\section{Hydroamination Products}

$\boldsymbol{N}$-(1-p-Tolylethyl)benzamide (2). ${ }^{2}$ A solution of benzamide (121 mg, $\left.1.0 \mathrm{mmol}\right), 4$ methylstyrene $(0.53 \mathrm{~mL}, 4.0 \mathrm{mmol}), 1(15 \mathrm{mg}, 0.025 \mathrm{mmol})$, and $\mathrm{P}\left(4-\mathrm{C}_{6} \mathrm{H}_{4} \mathrm{CF}_{3}\right)_{3}(23 \mathrm{mg}$, $0.050 \mathrm{mmol})$ in mesitylene $(0.5 \mathrm{~mL})$ was heated at $140{ }^{\circ} \mathrm{C}$ for $24 \mathrm{~h}$, cooled to room temperature, and chromatographed $\left(\mathrm{SiO}_{2} ;\right.$ hexanes-ethyl acetate $\left.=10: 1 \rightarrow 3: 1\right)$ to give 2 (204 $\mathrm{mg}, 85 \%)$ as a white solid. In a separate, identical experiment, the crude reaction mixture was cooled to room temperature and chromatographed $\left(\mathrm{SiO}_{2}\right.$; hexanes) to give $(E)$ 1,3-bis( $p$-tolyl)-1-butene (65 mg, 14\% based on 4-methylstyrene) as a colorless oil, which was identified by comparison to an authentic sample. ${ }^{3}$

For 2: $\mathrm{mp} 119-120{ }^{\circ} \mathrm{C}$. TLC: $\mathrm{R}_{f}=0.55 .{ }^{1} \mathrm{H}$ NMR: $\delta$ 7.77-7.75 (m, $\left.2 \mathrm{H}\right), 7.51-7.47$ (m, $1 \mathrm{H}), 7.44-7.40(\mathrm{~m}, 2 \mathrm{H}), 7.29$ (d, $J=8.0 \mathrm{~Hz}, 2 \mathrm{H}), 7.17(\mathrm{~d}, J=8.0 \mathrm{~Hz}, 2 \mathrm{H}), 6.29$ (br d, $J$ 
$=6.4 \mathrm{~Hz}, 1 \mathrm{H}), 5.31$ (quintet, $J=7.2 \mathrm{~Hz}, 1 \mathrm{H}), 2.34(\mathrm{~s}, 3 \mathrm{H}), 1.60(\mathrm{~d}, J=6.8 \mathrm{~Hz}, 3 \mathrm{H})$.

${ }^{13} \mathrm{C}\left\{{ }^{1} \mathrm{H}\right\}$ NMR: $\delta$ 166.8, 140.5, 137.5, 131.8, 129.8, 128.9, 127.2, 126.6, 49.3, 22.0, 21.4.

All remaining hydroamination reactions were performed employing a procedure analogous to that used to synthesize $\mathbf{2}$ unless noted otherwise. Reaction temperatures and yields are provided in Table 2 .

$N$-(1-Phenylethyl)benzamide (Table 2, entry 1). ${ }^{4}$ Reaction time $=45 \mathrm{~h} .{ }^{1} \mathrm{H}$ NMR: $\delta 7.75(\mathrm{~d}, J=8.0 \mathrm{~Hz}, 2 \mathrm{H}), 7.48-7.23(\mathrm{~m}, 8 \mathrm{H}), 6.35$ (br s, $1 \mathrm{H}$ ), 5.32 (quintet, $J=6.8 \mathrm{~Hz}, 1$ H), $1.58(\mathrm{~d}, J=6.8 \mathrm{~Hz}, 3 \mathrm{H}) .{ }^{13} \mathrm{C}\left\{{ }^{1} \mathrm{H}\right\} \mathrm{NMR}: \delta 166.9,143.5,134.9,131.8,129.1,128.9$, $127.8,127.3,126.6,49.5,22.1$.

$N$-[1-(4-Chlorophenyl)ethyl]benzamide (Table 2, entry 2). ${ }^{5}$ Reaction time $=72 \mathrm{~h}$. ${ }^{1} \mathrm{H}$ NMR: $\delta$ 7.74-7.72 (m, 2 H), 7.50-7.46 (m, $\left.1 \mathrm{H}\right), 7.42-7.38$ (m, $\left.2 \mathrm{H}\right), 7.29(\mathrm{~s}, 4 \mathrm{H}), 6.25$ (br s, $1 \mathrm{H}$ ), 5.28 (quintet, $J=6.8 \mathrm{~Hz}, 1 \mathrm{H}), 1.56(\mathrm{~d}, J=6.8 \mathrm{~Hz}, 3 \mathrm{H}) .{ }^{13} \mathrm{C}\left\{{ }^{1} \mathrm{H}\right\} \mathrm{NMR}: \delta$ $166.9,142.1,134.7,133.5,131.9,129.2,128.9,128.0,127.2,49.0,22.1$.

4-Methoxy- $N-(1-p$-tolylethyl)benzamide (Table 2, entry 3). Reaction time $=48 \mathrm{~h}$. White solid. mp 148-150 ${ }^{\circ} \mathrm{C}$. TLC: $\mathrm{R}_{f}=0.36 .{ }^{1} \mathrm{H}$ NMR: $\delta 7.70(\mathrm{~d}, J=8.4 \mathrm{~Hz}, 2 \mathrm{H}), 7.25$ (d, $J=8.0 \mathrm{~Hz}, 2 \mathrm{H}), 7.13(\mathrm{~d}, J=8.0 \mathrm{~Hz}, 2 \mathrm{H}), 6.87(\mathrm{~d}, J=8.4 \mathrm{~Hz}, 2 \mathrm{H}), 6.21$ (br d, $J=7.2$ $\mathrm{Hz}, 1 \mathrm{H}), 5.26$ (quintet, $J=7.2 \mathrm{~Hz}, 1 \mathrm{H}), 3.81(\mathrm{~s}, 3 \mathrm{H}), 2.31(\mathrm{~s}, 3 \mathrm{H}), 1.55(\mathrm{~d}, J=6.8 \mathrm{~Hz}, 3$ H). ${ }^{13} \mathrm{C}\left\{{ }^{1} \mathrm{H}\right\}$ NMR: $\delta \quad 166.3,162.5,140.7,137.4,129.7,129.0,127.3,126.5,114.0,55.7$, 49.2, 22.1, 21.4. IR (neat, $\mathrm{cm}^{-1}$ ): $3326(\mathrm{~m}), 1632$ (s). Anal. Calcd (found) for $\mathrm{C}_{17} \mathrm{H}_{19} \mathrm{NO}_{2}$ : C, 75.81 (75.59); H, 7.11 (7.10); N, 5.20 (5.23).

4-Bromo- $N$-(1-p-tolylethyl)benzamide (Table 2, entry 4). Reaction time $=24 \mathrm{~h}$. White solid. mp 150-151 ${ }^{\circ} \mathrm{C}$. TLC: $\mathrm{R}_{f}=0.57 .{ }^{1} \mathrm{H}$ NMR: $\delta 7.58(\mathrm{~d}, J=7.6 \mathrm{~Hz}, 2 \mathrm{H}), 7.47$ (d, $J=7.6 \mathrm{~Hz}, 2 \mathrm{H}), 7.22(\mathrm{~d}, J=6.0 \mathrm{~Hz}, 2 \mathrm{H}), 7.12(\mathrm{~d}, J=7.2 \mathrm{~Hz}, 2 \mathrm{H}), 6.52($ br s, $1 \mathrm{H}), 5.22$ 
(quintet, $J=6.8 \mathrm{~Hz}, 1 \mathrm{H}), 2.31(\mathrm{~s}, 3 \mathrm{H}), 1.54(\mathrm{~d}, J=6.4 \mathrm{~Hz}, 3 \mathrm{H}) .{ }^{13} \mathrm{C}\left\{{ }^{1} \mathrm{H}\right\} \mathrm{NMR}: \delta 165.9$, $140.3,137.5,133.7,132.0,129.7,128.9,126.5,126.3,49.4,21.9,21.4$. IR (neat, $\mathrm{cm}^{-1}$ ): 3300 (m), 1638 (s). Anal. Calcd (found) for $\mathrm{C}_{16} \mathrm{H}_{16} \mathrm{BrNO}$ : C, 60.39 (60.26); H, 5.07 (4.85); N, 4.40 (4.13).

$N$-(1-p-Tolylethyl)-4-trifluoromethylbenzamide (Table 2, entry 5). Reaction time $=24 \mathrm{~h}$. White solid. $\mathrm{mp} 143-144{ }^{\circ} \mathrm{C}$. TLC: $\mathrm{R}_{f}=0.69 .{ }^{1} \mathrm{H}$ NMR: $\delta 7.83(\mathrm{~d}, J=8.0 \mathrm{~Hz}, 2$ H), 7.64 (d, $J=8.4 \mathrm{~Hz}, 2 \mathrm{H}), 7.25$ (d, $J=8.0 \mathrm{~Hz}, 2 \mathrm{H}), 7.15$ (d, $J=7.6 \mathrm{~Hz}, 2 \mathrm{H}), 6.34$ (br d, $J$ $=6.8 \mathrm{~Hz}, 1 \mathrm{H}), 5.27$ (quintet, $J=6.8 \mathrm{~Hz}, 1 \mathrm{H}), 2.32(\mathrm{~s}, 3 \mathrm{H}), 1.58(\mathrm{~d}, J=6.8 \mathrm{~Hz}, 3 \mathrm{H})$. ${ }^{13} \mathrm{C}\left\{{ }^{1} \mathrm{H}\right\}$ NMR: $\delta \quad 165.7,140.2,138.2,137.6,133.4\left(\mathrm{q},{ }^{2} J_{\mathrm{CF}}=32 \mathrm{~Hz}\right), 129.7,127.8,126.5$, $125.8\left(\mathrm{q},{ }^{3} J_{\mathrm{CF}}=3.5 \mathrm{~Hz}\right), 124.0\left(\mathrm{q},{ }^{1} J_{\mathrm{CF}}=271 \mathrm{~Hz}\right), 49.6,21.9,21.3 .{ }^{19} \mathrm{~F}$ NMR: $\delta-63.4$. IR (neat, $\mathrm{cm}^{-1}$ ): 3289 (m), 1643 (s). Anal. Calcd (found) for $\mathrm{C}_{17} \mathrm{H}_{16} \mathrm{~F}_{3} \mathrm{NO}$ : C, 66.44 (66.26); H, $5.25(5.32) ; \mathrm{N}, 4.56(4.84)$.

Methyl $N$-(1-p-tolylethyl)terephthalamate (Table 2, entry 6). Reaction time $=30$ h. White solid. mp $153-154{ }^{\circ} \mathrm{C}$. TLC: $\mathrm{R}_{f}=0.30 .{ }^{1} \mathrm{H}$ NMR (Figure S1): $\delta 8.07(\mathrm{~d}, J=8.8$ Hz, 2 H), 7.81 (d, $J=8.8 \mathrm{~Hz}, 2 \mathrm{H}), 7.29(\mathrm{~d}, J=8.0 \mathrm{~Hz}, 2 \mathrm{H}), 7.18$ (d, $J=8.0 \mathrm{~Hz}, 2 \mathrm{H}), 6.37$ (d, $J=7.8 \mathrm{~Hz}, 1 \mathrm{H}), 5.30$ (quintet, $J=6.8 \mathrm{~Hz}, 1 \mathrm{H}), 3.93(\mathrm{~s}, 3 \mathrm{H}), 2.34(\mathrm{~s}, 3 \mathrm{H}), 1.61(\mathrm{~d}, J=$ $6.8 \mathrm{~Hz}, 3 \mathrm{H}$ ). ${ }^{13} \mathrm{C}\left\{{ }^{1} \mathrm{H}\right\}$ NMR (Figure S2): $\delta$ 166.6, 166.0, 140.1, 138.9, 137.7, 133.0, 130.1, 129.8, 127.3, 126.6, 52.7, 49.6, 21.9, 21.4. IR (neat, $\mathrm{cm}^{-1}$ ): 3294 (br s), 1723 (s), 1635 (s), 1538 (s), 1275 (s). HRMS Calcd (found) for $\mathrm{C}_{18} \mathrm{H}_{19} \mathrm{NO}_{3}$ : 297.1365 (297.1372).

4-Methyl- $N$-(1-p-tolylethyl)benzamide (Table 2, entry 7). Reaction time $=24 \mathrm{~h}$. White solid. mp 116-118 ${ }^{\circ} \mathrm{C}$. TLC: $\mathrm{R}_{f}=0.56 .{ }^{1} \mathrm{H}$ NMR: $\delta 7.66(\mathrm{~d}, J=8.0 \mathrm{~Hz}, 2 \mathrm{H}), 7.29$ (d, $J=8.0 \mathrm{~Hz}, 2 \mathrm{H}), 7.21(\mathrm{~d}, J=8.0 \mathrm{~Hz}, 2 \mathrm{H}), 7.17(\mathrm{~d}, J=8.0 \mathrm{~Hz}, 2 \mathrm{H}), 6.28$ (br d, $J=7.8$ $\mathrm{Hz}, 1 \mathrm{H}), 5.30$ (quintet, $J=6.8 \mathrm{~Hz}, 1 \mathrm{H}), 2.39$ (s, $3 \mathrm{H}), 2.34$ (s, $3 \mathrm{H}), 1.59$ (d, $J=6.8 \mathrm{~Hz}, 3$ 
H). ${ }^{13} \mathrm{C}\left\{{ }^{1} \mathrm{H}\right\}$ NMR: $\delta 166.8,142.1,140.6,137.4,132.1,129.7,129.5,127.2,126.5,49.2$, 22.0, 21.8, 21.4. IR (neat, $\mathrm{cm}^{-1}$ ): 3305 (m), 1638 (s). Anal. Calcd (found) for $\mathrm{C}_{17} \mathrm{H}_{19} \mathrm{NO}$ : C, 80.60 (80.36); H, 7.56 (7.32); N, 5.53 (4.99).

2-Methyl- $N$-(1-p-tolylethyl)benzamide (Table 2, entry 8). Reaction time $=48 \mathrm{~h}$. White solid. mp $126-127{ }^{\circ} \mathrm{C}$. TLC: $\mathrm{R}_{f}=0.61 .{ }^{1} \mathrm{H}$ NMR: $\delta$ 7.32-7.24 $(\mathrm{m}, 4 \mathrm{H}), 7.18-7.13$ (m, $4 \mathrm{H}), 5.91$ (br s, $1 \mathrm{H}), 5.27$ (quintet, $J=6.8 \mathrm{~Hz}, 1 \mathrm{H}), 2.40(\mathrm{~s}, 3 \mathrm{H}), 2.32(\mathrm{~s}, 3 \mathrm{H}), 1.55(\mathrm{~d}$, $J=6.8 \mathrm{~Hz}, 3 \mathrm{H}) .{ }^{13} \mathrm{C}\left\{{ }^{1} \mathrm{H}\right\}$ NMR: $\delta 169.4,140.4,137.5,136.4,131.3,130.1,129.7,126.9$, 126.5, 126.0, 49.1, 22.1, 21.4, 20.1. IR (neat, $\mathrm{cm}^{-1}$ ): 3258 (m), 1638 (s). Anal. Calcd (found) for $\mathrm{C}_{17} \mathrm{H}_{19} \mathrm{NO}: \mathrm{C}, 80.60$ (80.48); $\mathrm{H}, 7.56$ (7.51); N, 5.53 (5.79).

$N$-(1-p-Tolylethyl)-2-napthylcarboxamide (Table 2, entry 9). Reaction time $=24$ h. White solid. mp 131-133 ${ }^{\circ} \mathrm{C}$. TLC: $\mathrm{R}_{f}=0.62 .{ }^{1} \mathrm{H}$ NMR: $\delta 8.28(\mathrm{~s}, 1 \mathrm{H}), 7.89-7.82(\mathrm{~m}$, $4 \mathrm{H}), 7.57-7.50(\mathrm{~m}, 2 \mathrm{H}), 7.33(\mathrm{~d}, J=8.0 \mathrm{~Hz}, 2 \mathrm{H}), 7.18(\mathrm{~d}, J=8.0 \mathrm{~Hz}, 2 \mathrm{H}), 6.60$ (br d, $J=$ $7.2 \mathrm{~Hz}, 1 \mathrm{H}), 5.37$ (quintet, $J=6.8 \mathrm{~Hz}, 1 \mathrm{H}), 2.35(\mathrm{~s}, 3 \mathrm{H}), 1.64(\mathrm{~d}, J=6.8 \mathrm{~Hz}, 3 \mathrm{H}) .{ }^{13} \mathrm{C}\left\{{ }^{1} \mathrm{H}\right\}$ NMR: $\delta 166.9,140.5,137.5,135.0,132.9,132.2,129.7,129.2,128.7,128.0,127.9,127.6$, 127.0, 126.6, 124.0, 49.4, 22.0, 21.4. IR (neat, $\mathrm{cm}^{-1}$ ): 3300 (m), 1638 (s). Anal. Calcd (found) for $\mathrm{C}_{20} \mathrm{H}_{19} \mathrm{NO}$ : $\mathrm{C}, 83.01$ (82.87); H, 6.62 (6.55); N, 4.84 (5.02).

$N$-[1-(p-tolyl)ethyl]-benzo[1,3]dioxole-5-carboxamide (Table 2, entry 10). Reaction time $=24$ h. Pale yellow solid. mp $137-138^{\circ} \mathrm{C}$. TLC: $R_{f}=0.39 .{ }^{1} \mathrm{H}$ NMR (Figure S3): $\delta$ 7.26-7.22 (m, $4 \mathrm{H}), 7.12(\mathrm{~d}, J=8.0 \mathrm{~Hz}, 2 \mathrm{H}), 6.75(\mathrm{~d}, J=8.0 \mathrm{~Hz}, 1 \mathrm{H}), 6.31(\mathrm{~d}, J=$ $7.2 \mathrm{~Hz}, 1 \mathrm{H}), 5.96$ (s, $2 \mathrm{H}), 5.22$ (quintet, $J=6.8 \mathrm{~Hz}, 1 \mathrm{H}), 2.30(\mathrm{~s}, 3 \mathrm{H}), 1.53(\mathrm{~d}, J=6.8 \mathrm{~Hz}$, $3 \mathrm{H}) .{ }^{13} \mathrm{C}\left\{{ }^{1} \mathrm{H}\right\}$ NMR (Figure S4): $\delta$ 166.1, 150.5, 148.2, 140.6, 137.4, 129.7, 129.2, 126.5, 121.8, 108.2, 108.0, 101.9, 49.3, 22.0, 21.3. IR (neat, $\mathrm{cm}^{-1}$ ): 3435 (br s), 1635 (s), 1479 (s), 1256 (s). HRMS Calcd (found) for $\mathrm{C}_{17} \mathrm{H}_{17} \mathrm{NO}_{3}: 283.1208$ (283.1205). 
$N$-[1-(o-tolyl)ethyl]benzamide (Table 2, entry 11). Reaction time $=72 \mathrm{~h} . \quad$ Pale yellow solid. mp 130-131 ${ }^{\circ} \mathrm{C}$. TLC: $\mathrm{R}_{f}=0.33 .{ }^{1} \mathrm{H}$ NMR (Figure S5): $\delta 7.76(\mathrm{~d}, J=8.4$ Hz, $2 \mathrm{H}), 7.49(7, J=7.6 \mathrm{~Hz}, 1 \mathrm{H}), 7.43-7.37$ (m, $3 \mathrm{H}), 7.26-7.19$ (m, $3 \mathrm{H}), 6.31$ (br s, $1 \mathrm{H})$, 5.51 (quintet, $J=7.2 \mathrm{~Hz}, 1 \mathrm{H}), 2.43(\mathrm{~s}, 3 \mathrm{H}), 1.59(\mathrm{~d}, J=6.8 \mathrm{~Hz}, 3 \mathrm{H}) .{ }^{13} \mathrm{C}\left\{{ }^{1} \mathrm{H}\right\} \mathrm{NMR}$ (Figure S6): $\delta$ 166.7, 141.3, 136.5, 134.9, 131.8, 131.2, 128.9, 127.8, 127.2, 126.7, 125.1, 46.2, 21.4, 19.5. IR (neat, $\mathrm{cm}^{-1}$ ): 3299 (m), 1630 (s). HRMS Calcd (found) for $\mathrm{C}_{16} \mathrm{H}_{17} \mathrm{NO}$ $\left(\mathrm{M}^{+}\right): 239.1310(239.1315)$.

$N$-[1-(2,4-Dimethylphenyl)ethyl]benzamide $($ Table 2 , entry 12$) .{ }^{6}$ Reaction time $=$ 24 h. ${ }^{1} \mathrm{H}$ NMR: $\delta 7.73(\mathrm{~d}, J=8.0 \mathrm{~Hz}, 2 \mathrm{H}), 7.45(\mathrm{t}, J=8.0 \mathrm{~Hz}, 1 \mathrm{H}), 7.37(\mathrm{~d}, J=8.0 \mathrm{~Hz}, 2$ H), $7.25(\mathrm{~d}, J=8.0 \mathrm{~Hz}, 1 \mathrm{H}), 7.01(\mathrm{~d}, J=9.2 \mathrm{~Hz}, 2 \mathrm{H}), 6.38(\mathrm{br} \mathrm{s}, 1 \mathrm{H}), 5.45$ (quintet, $J=6.8$ $\mathrm{Hz}, 1 \mathrm{H}), 2.36$ (s, $3 \mathrm{H}), 2.29(\mathrm{~s}, 3 \mathrm{H}), 1.55(\mathrm{~d}, J=6.8 \mathrm{~Hz}, 3 \mathrm{H}) .{ }^{13} \mathrm{C}\left\{{ }^{1} \mathrm{H}\right\} \mathrm{NMR}: \delta$ 166.6, $138.4,137.3,136.3,134.8,131.9,131.6,128.8,127.2,125.1,45.9,21.3,21.2,19.4$.

4-(tert-Butyldimethylsiloxy)- $N$-[1-(2,4-dimethylphenyl)ethyl]benzamide (Table 2, entry 13). Reaction time $=44 \mathrm{~h}$. Pale yellow solid. mp 103-105 ${ }^{\circ} \mathrm{C}$. TLC: $\mathrm{R}_{f}=0.64 .{ }^{1} \mathrm{H}$ NMR: $\delta 7.62(\mathrm{~d}, J=8.4 \mathrm{~Hz}, 2 \mathrm{H}), 7.23(\mathrm{~d}, J=8.0 \mathrm{~Hz}, 1 \mathrm{H}), 7.01(\mathrm{~d}, J=8.0 \mathrm{~Hz}, 1 \mathrm{H}), 6.99$ (s, $1 \mathrm{H}), 6.80(\mathrm{~d}, J=8.8 \mathrm{~Hz}, 2 \mathrm{H}), 6.08(\mathrm{br} \mathrm{d}, J=7.6 \mathrm{~Hz}, 1 \mathrm{H}$ ), 5.42 (quintet, $J=6.8 \mathrm{~Hz}, 1 \mathrm{H}$ ), $2.35(\mathrm{~s}, 3 \mathrm{H}), 2.28(\mathrm{~s}, 3 \mathrm{H}), 1.54(\mathrm{~d}, J=6.4 \mathrm{~Hz}, 3 \mathrm{H}), 0.94(\mathrm{~s}, 9 \mathrm{H}), 0.17(\mathrm{~s}, 6 \mathrm{H}) .{ }^{13} \mathrm{C}\left\{{ }^{1} \mathrm{H}\right\}$ NMR: $\delta 166.3,159.0,138.5,137.3,136.4,131.9,128.9,127.8,127.2,125.1,120.3,45.9$, 25.9, 21.35, 21.26, 19.4, 18.6, -4.1. IR (neat, $\left.\mathrm{cm}^{-1}\right)$ : $3310(\mathrm{~m}), 1638$ (s). Anal. Calcd (found) for $\mathrm{C}_{23} \mathrm{H}_{33} \mathrm{NO}_{2} \mathrm{Si}$ : C, 72.01 (71.90); $\mathrm{H}, 8.67$ (8.94); N, 3.65 (4.00).

$N$-(1-Indanyl)benzamide (Table 2, entry 14). ${ }^{7}$ Reaction time $=48 \mathrm{~h} .{ }^{1} \mathrm{H}$ NMR: $\delta$ 7.76-7.74 (m, 2 H), 7.48-7.44 (m, $1 \mathrm{H}), 7.40-7.36$ (m, $2 \mathrm{H}), 7.31$ (d, J= 7.2 Hz, $1 \mathrm{H})$, 7.247.17 (m, $3 \mathrm{H}), 6.53$ (br d, $J=7.6 \mathrm{~Hz}, 1 \mathrm{H}), 5.63$ (q, $J=7.6 \mathrm{~Hz}, 1 \mathrm{H}), 2.98$ (ddd, $J=4.0,8.8$, 
$16 \mathrm{~Hz}, 1 \mathrm{H}), 2.87$ (td, $J=8.0,16 \mathrm{~Hz}, 1 \mathrm{H}), 2.63(\mathrm{dtd}, J=3.6,6.8,16.8 \mathrm{~Hz}, 1 \mathrm{H}), 1.89$ (qd, $J=$ 8.4, $12.8 \mathrm{~Hz}, 1 \mathrm{H}) .{ }^{13} \mathrm{C}\left\{{ }^{1} \mathrm{H}\right\}$ NMR: $\delta 167.5,143.8,143.5,134.8,131.8,128.9,128.4,127.3$, $127.1,125.2,124.4,55.4,34.4,30.6$.

3-(1-p-Tolylethyl)-2-oxazolidone (Table 15). ${ }^{8}$ Reaction time $=48 \mathrm{~h} .{ }^{1} \mathrm{H}$ NMR: $\delta$ $7.17(\mathrm{~d}, J=8.0 \mathrm{~Hz}, 2 \mathrm{H}), 7.11(\mathrm{~d}, J=8.0 \mathrm{~Hz}, 2 \mathrm{H}), 5.12(\mathrm{q}, J=6.8 \mathrm{~Hz}, 1 \mathrm{H}), 4.23$ (q, $J=8.4$ Hz, $1 \mathrm{H}), 4.15(\mathrm{q}, J=8.8 \mathrm{~Hz}, 1 \mathrm{H}), 3.44(\mathrm{q}, J=8.4 \mathrm{~Hz}, 1 \mathrm{H}), 3.10$ (q, $J=8.4 \mathrm{~Hz}, 1 \mathrm{H}), 2.29$ (s, $3 \mathrm{H}), 1.51(\mathrm{~d}, J=7.2 \mathrm{~Hz}, 3 \mathrm{H}) .{ }^{13} \mathrm{C}\left\{{ }^{1} \mathrm{H}\right\} \mathrm{NMR}: \delta 158.2,137.7,136.8,129.5,127.1,62.1$, $51.4,40.2,21.2,16.6$.

$(N-1-p$-Tolylethyl)valeramide (Table 2, entry 16). Reaction time $=48 \mathrm{~h}$. Viscous pale yellow oil. TLC (hexanes-EtOAc $=1: 1): \mathrm{R}_{f}=0.45 .{ }^{1} \mathrm{H}$ NMR (Figure S7): $\delta 7.21(\mathrm{~d}$, $J=8.0 \mathrm{~Hz}, 2 \mathrm{H}), 7.14(\mathrm{~d}, J=8.0 \mathrm{~Hz}, 2 \mathrm{H}), 5.71(\mathrm{~d}, J=6.4 \mathrm{~Hz}, 1 \mathrm{H}), 5.10$ (quintet, $J=7.2$ $\mathrm{Hz}, 1 \mathrm{H}), 2.33(\mathrm{~s}, 3 \mathrm{H}), 2.16$ (t, $J=8.0 \mathrm{~Hz}, 2 \mathrm{H}), 1.61$ (quintet, $J=7.6 \mathrm{~Hz}, 2 \mathrm{H}), 1.46$ (d, $J=$ $6.8 \mathrm{~Hz}, 3 \mathrm{H}$ ), 1.33 (sextet, $J=7.2 \mathrm{~Hz}, 2 \mathrm{H}$ ), 0.90 (t, $J=7.2 \mathrm{~Hz}, 3 \mathrm{H}$ ). ${ }^{13} \mathrm{C}\left\{{ }^{1} \mathrm{H}\right\}$ NMR (Figure S8): $\delta 172.4,140.7,137.3,129.6,126.4,48.6,37.0,28.1,22.7,22.0,21.4,14.1$. IR (neat, $\mathrm{cm}^{-1}$ ): $3289(\mathrm{~m}), 1638$ (s). HRMS Calcd (found) for $\mathrm{C}_{14} \mathrm{H}_{21} \mathrm{NO}\left(\mathrm{M}^{+}\right): 219.1623$ (219.1628).

[N-1-(p-Methoxyphenyl)ethyl]valeramide (Table 2, entry 17). Reaction time $=48$ h. Viscous colorless oil. TLC (hexanes-EtOAc $=1: 1): \mathrm{R}_{f}=0.42 .{ }^{1} \mathrm{H}$ NMR (Figure S9): $\delta$ $7.23(\mathrm{~d}, J=8.8 \mathrm{~Hz}, 2 \mathrm{H}), 6.85(\mathrm{~d}, J=8.4 \mathrm{~Hz}, 2 \mathrm{H}), 5.80$ (br d, $J=6.8 \mathrm{~Hz}, 1 \mathrm{H}$ ), 5.08 (quintet, $J=7.2 \mathrm{~Hz}, 1 \mathrm{H}$ ), $3.78(\mathrm{~s}, 3 \mathrm{H}), 2.14$ (t, $J=7.6 \mathrm{~Hz}, 2 \mathrm{H}), 1.59$ (quintet, $J=7.6 \mathrm{~Hz}, 2 \mathrm{H}$ ), 1.45 $(\mathrm{d}, J=7.2 \mathrm{~Hz}, 3 \mathrm{H}), 1.31$ (sextet, $J=7.2 \mathrm{~Hz}, 2 \mathrm{H}), 0.89(\mathrm{t}, J=7.2 \mathrm{~Hz}, 3 \mathrm{H}) .{ }^{13} \mathrm{C}\left\{{ }^{1} \mathrm{H}\right\} \mathrm{NMR}$ (Figure S10): $\delta 172.4,159.0,135.8,127.7,114.2,55.6,48.2,36.9,28.1,22.7,21.9,14.1$. IR (neat, $\mathrm{cm}^{-1}$ ): $3284(\mathrm{~m}), 1644$ (s). HRMS Calcd (found) for $\mathrm{C}_{14} \mathrm{H}_{22} \mathrm{NO}_{2}\left(\mathrm{MH}^{+}\right): 236.1651$ (236.1645). 
$N$-(1-p-Tolylethyl)-p-toluenesulfonamide (Table 2, entry 18). ${ }^{9}$ Reaction time $=24$

h. ${ }^{1} \mathrm{H}$ NMR: $\delta 7.63(\mathrm{~d}, J=8.0 \mathrm{~Hz}, 2 \mathrm{H}), 7.18(\mathrm{~d}, J=8.0 \mathrm{~Hz}, 2 \mathrm{H}), 6.99(\mathrm{~s}, 4 \mathrm{H}), 4.98(\mathrm{~d}, J=$ $6.8 \mathrm{~Hz}, 1 \mathrm{H}), 4.41$ (quintet, $J=6.8 \mathrm{~Hz}, 1 \mathrm{H}), 2.39(\mathrm{~s}, 3 \mathrm{H}), 2.28(\mathrm{~s}, 3 \mathrm{H}), 1.40(\mathrm{~d}, J=6.8 \mathrm{~Hz}$, $3 \mathrm{H}) .{ }^{13} \mathrm{C}\left\{{ }^{1} \mathrm{H}\right\}$ NMR: $\delta 143.4,139.4,138.0,137.5,129.7,129.5,127.4,126.4,53.7,23.8$, 21.8, 21.3. 
Figure S1. ${ }^{1} \mathrm{H}$ NMR spectrum of methyl $N$-(1-p-tolylethyl)terephthalamate.

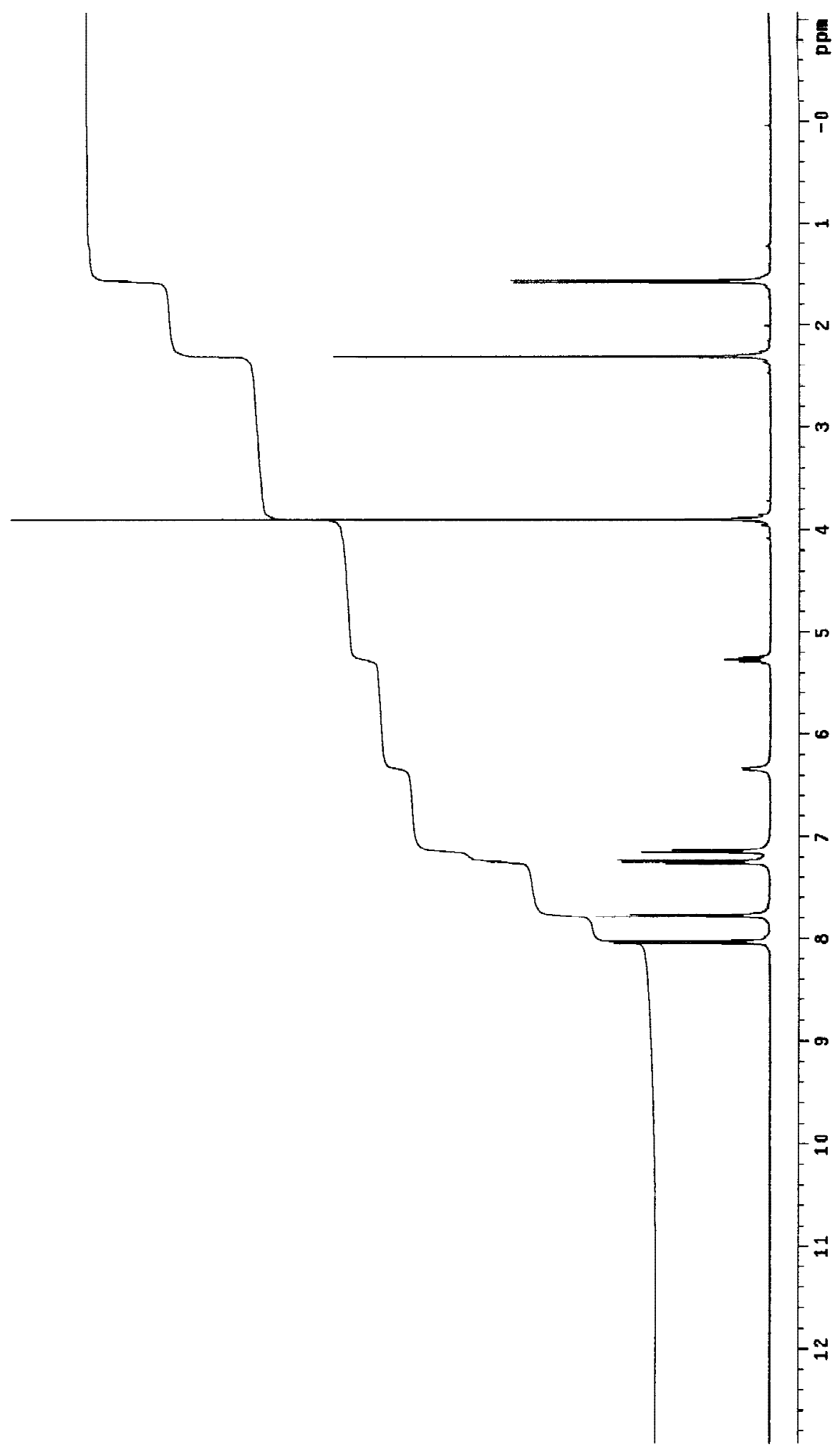


Figure S2. ${ }^{13} \mathrm{C}\left\{{ }^{1} \mathrm{H}\right\}$ NMR spectrum of methyl $N$-(1-p-tolylethyl)terephthalamate.

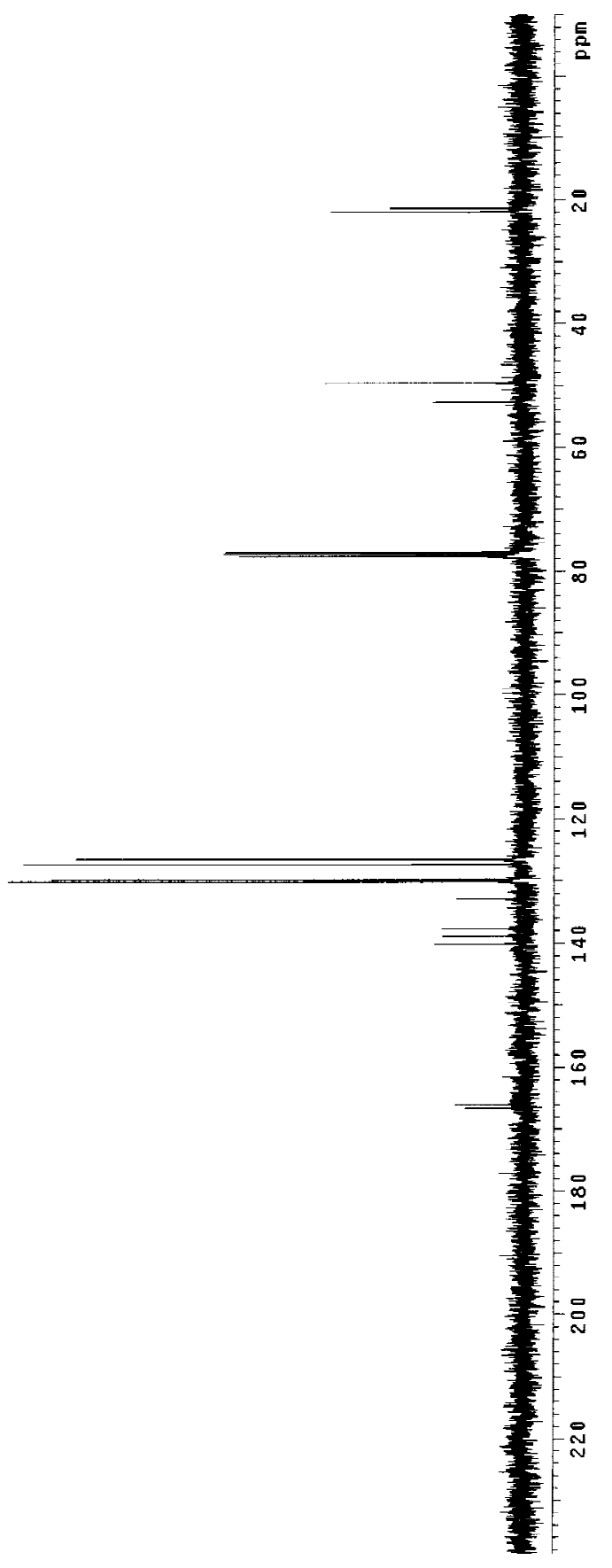


Figure S3. ${ }^{1} \mathrm{H}$ NMR spectrum of $N$-[1-( $p$-tolyl)ethyl]-benzo[1,3]dioxole-5-carboxamide.

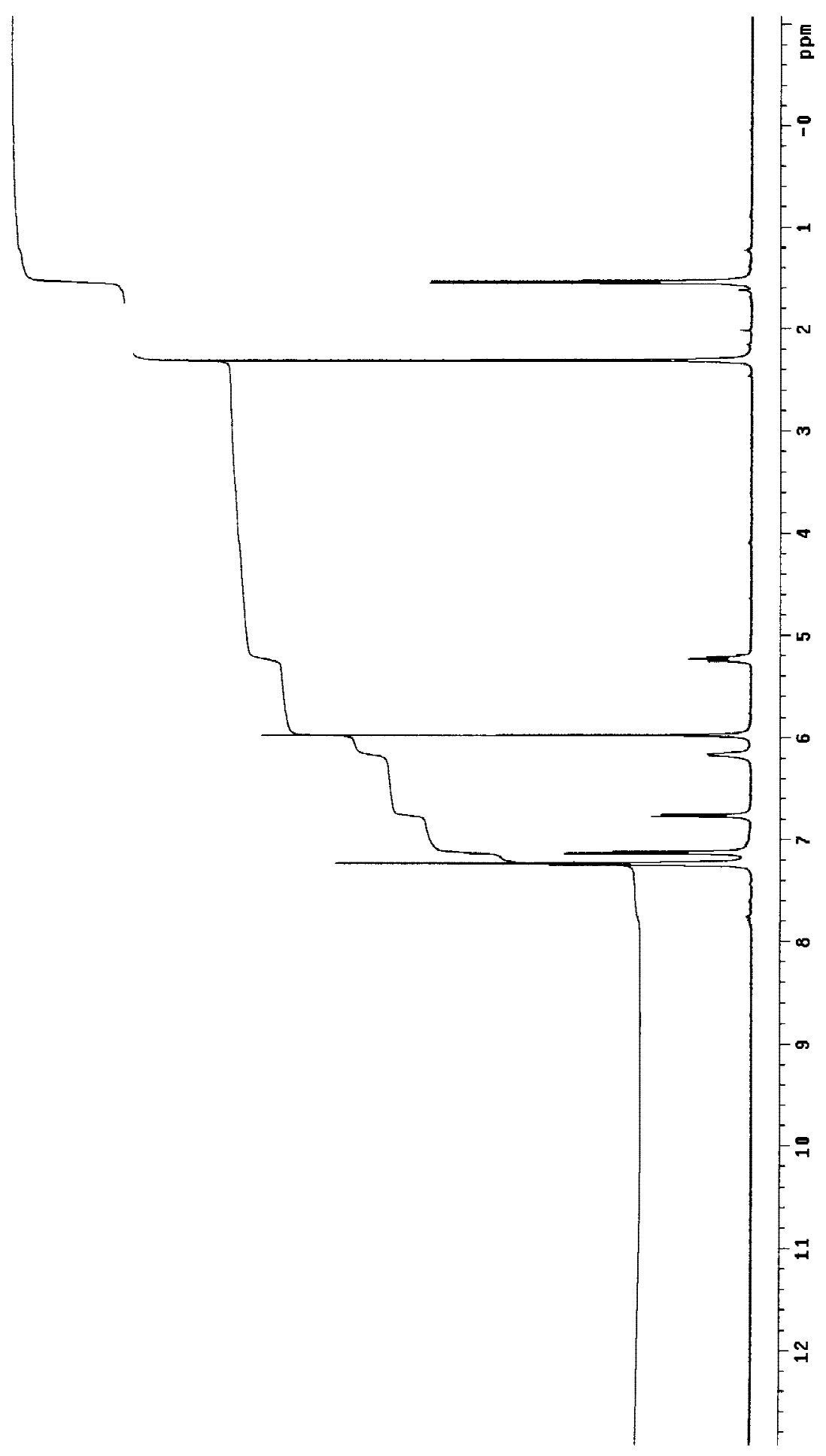


Figure S4. ${ }^{13} \mathrm{C}\left\{{ }^{1} \mathrm{H}\right\} \quad \mathrm{NMR}$ spectrum of $N$-[1-(p-tolyl)ethyl]-benzo[1,3]dioxole-5carboxamide.

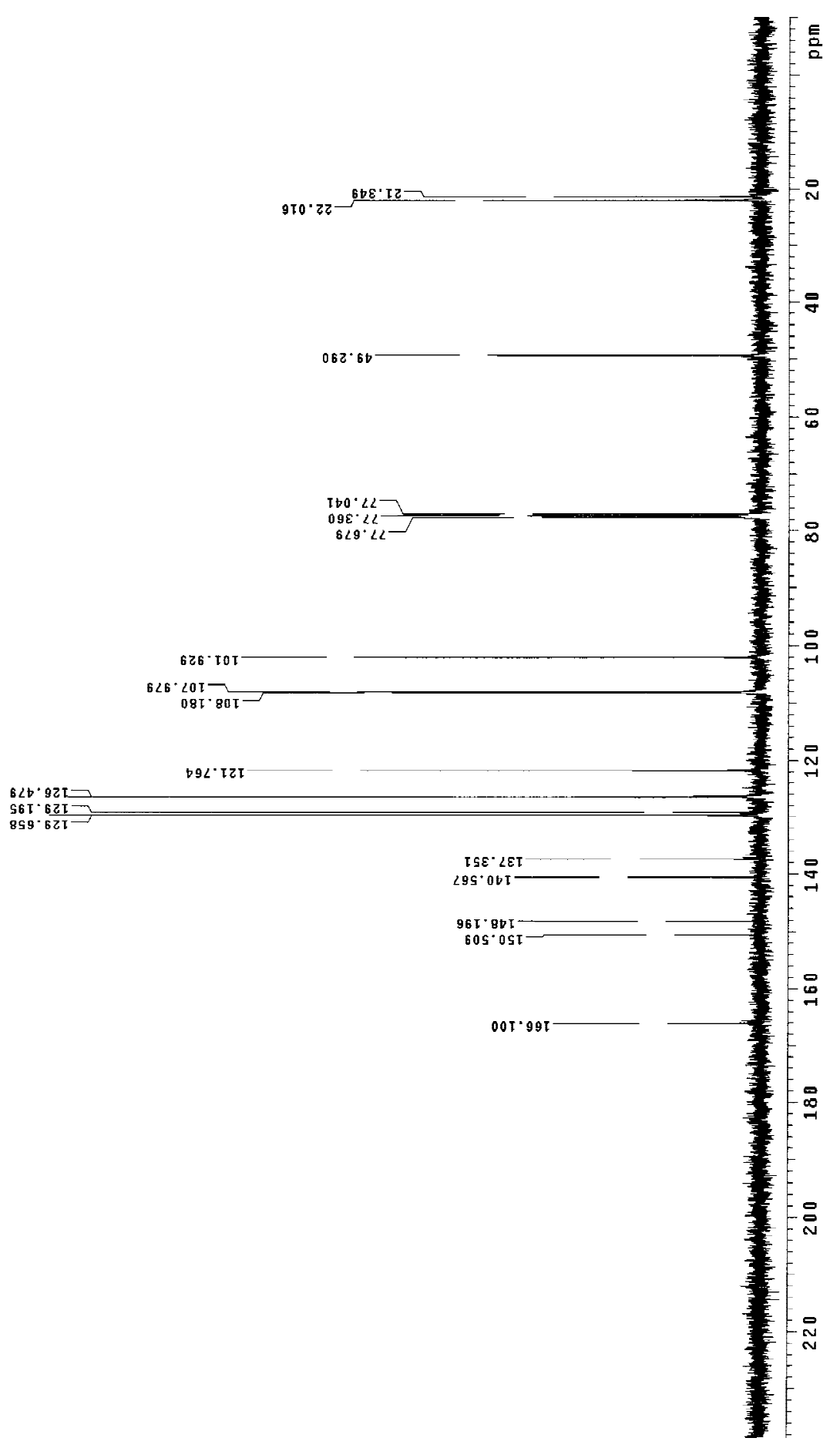


Figure S5. ${ }^{1} \mathrm{H}$ NMR spectrum of $N$-[1-(o-tolyl)ethyl]benzamide.

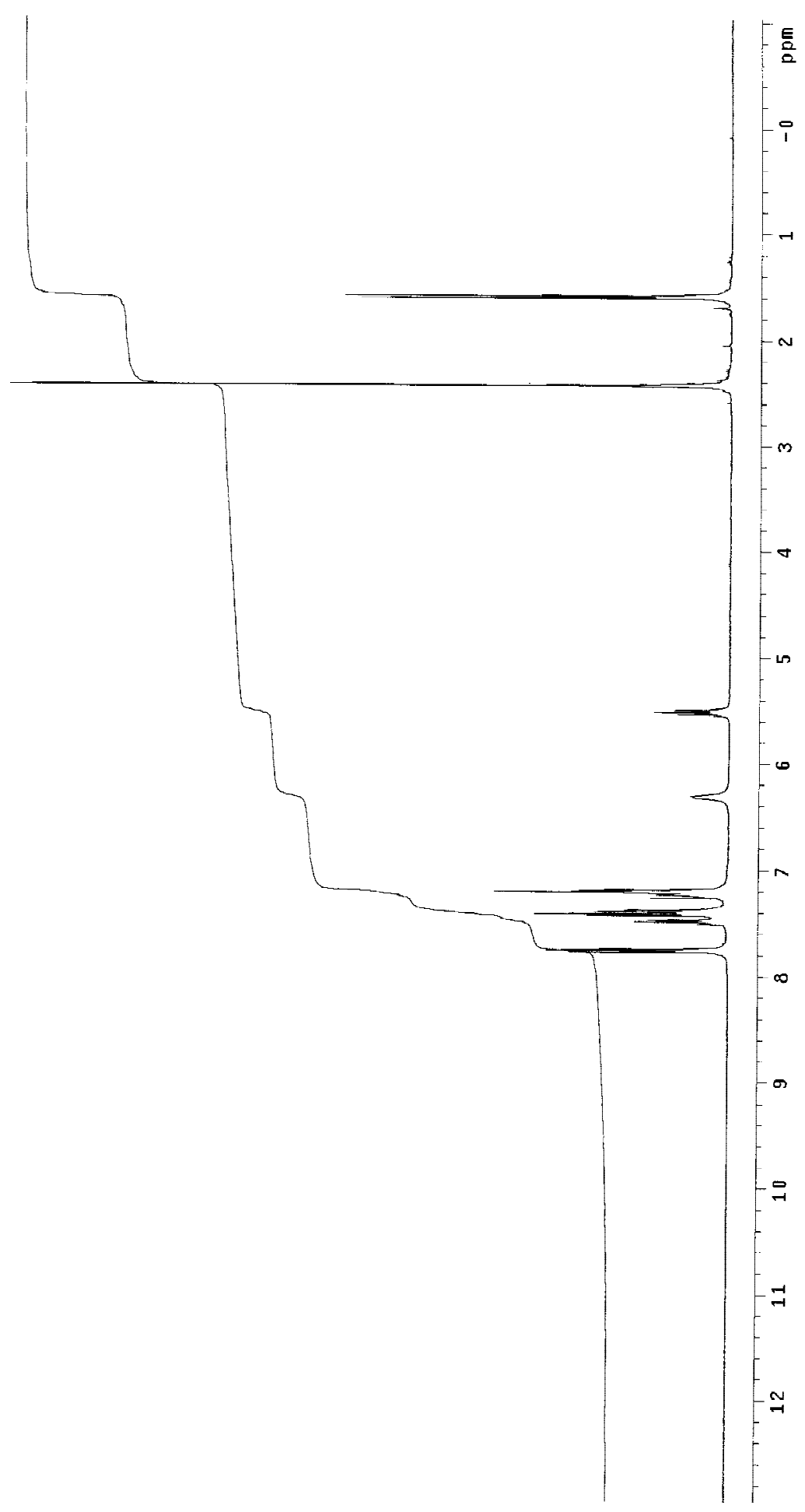


Figure S6. ${ }^{13} \mathrm{C}\left\{{ }^{1} \mathrm{H}\right\}$ NMR spectrum of $N$-[1-(o-tolyl)ethyl]benzamide.

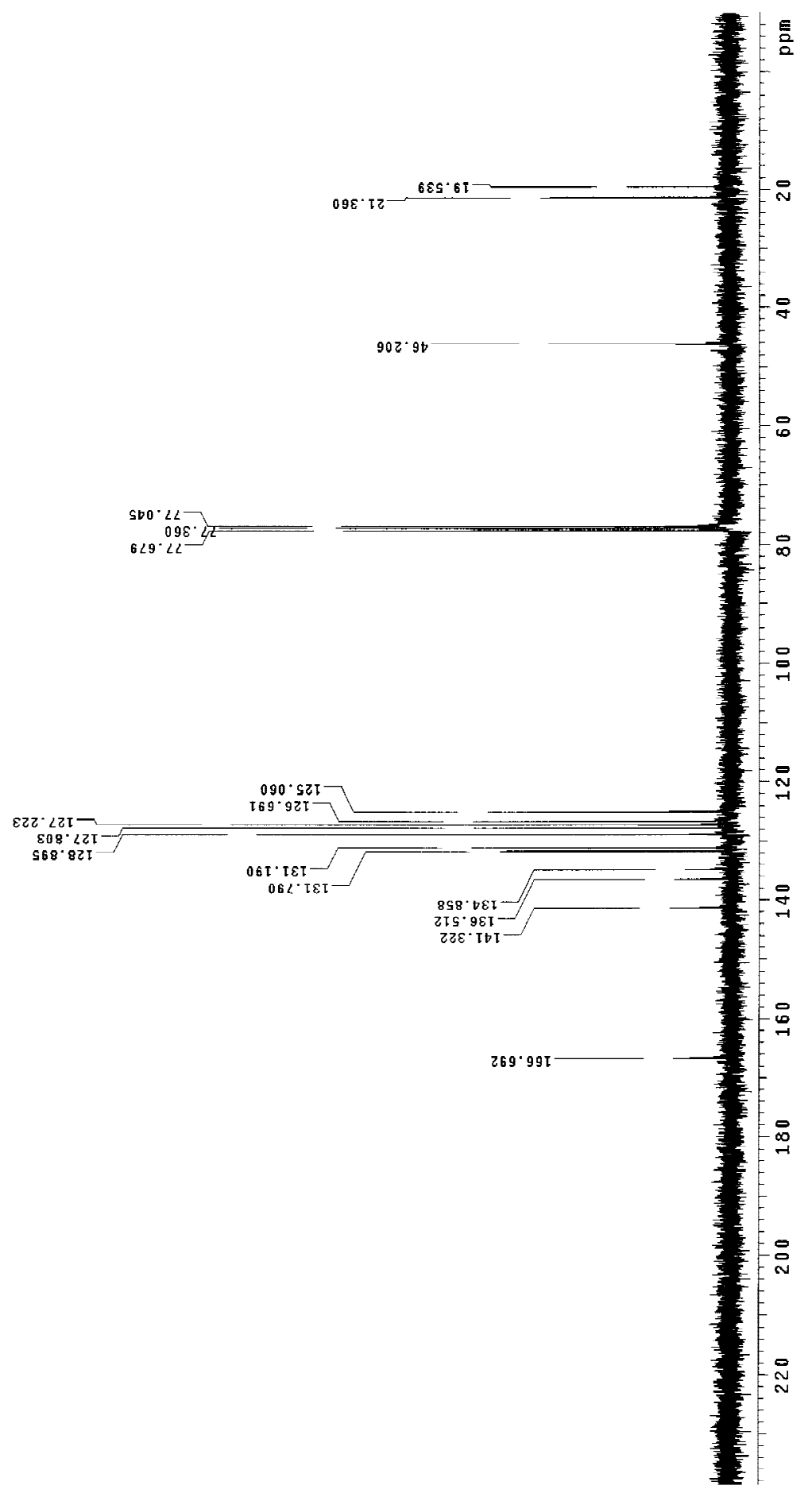


Figure S7. ${ }^{1} \mathrm{H}$ NMR spectrum of ( $N-1-p$-tolylethyl)valeramide.

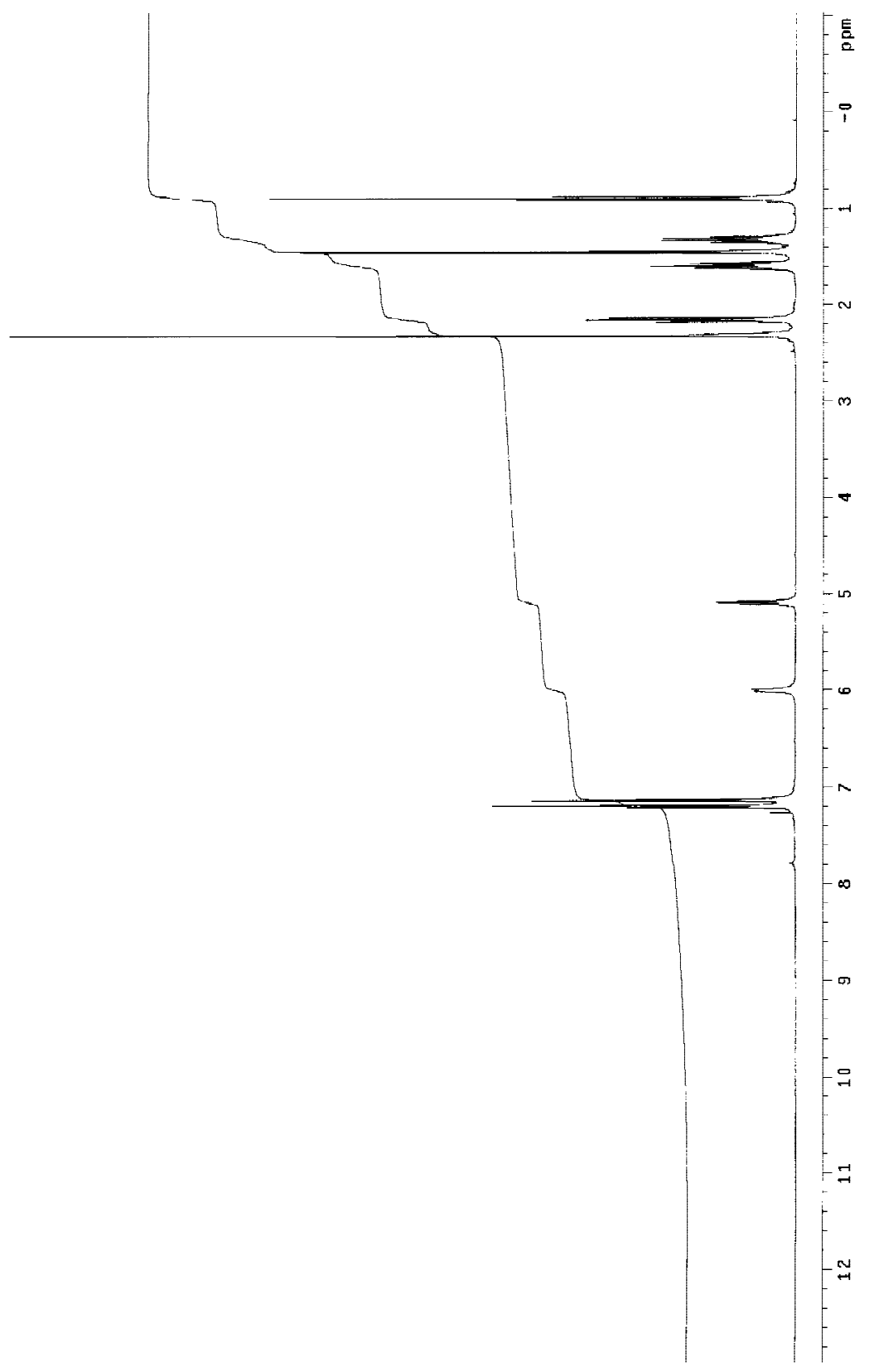


Figure S8. ${ }^{13} \mathrm{C}\left\{{ }^{1} \mathrm{H}\right\}$ NMR spectrum of $(N-1-p$-tolylethyl)valeramide.

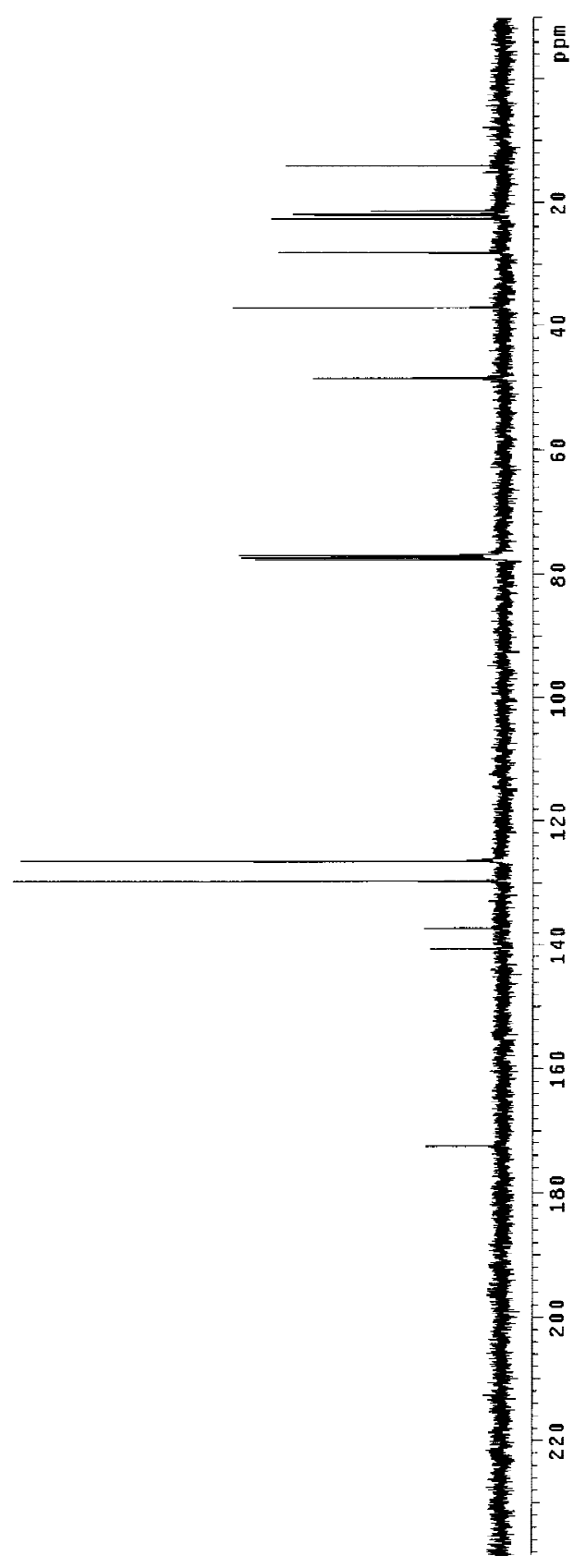


Figure S9. ${ }^{1} \mathrm{H}$ NMR spectrum of [ $N-1-(p$-methoxyphenyl)ethyl]valeramide.

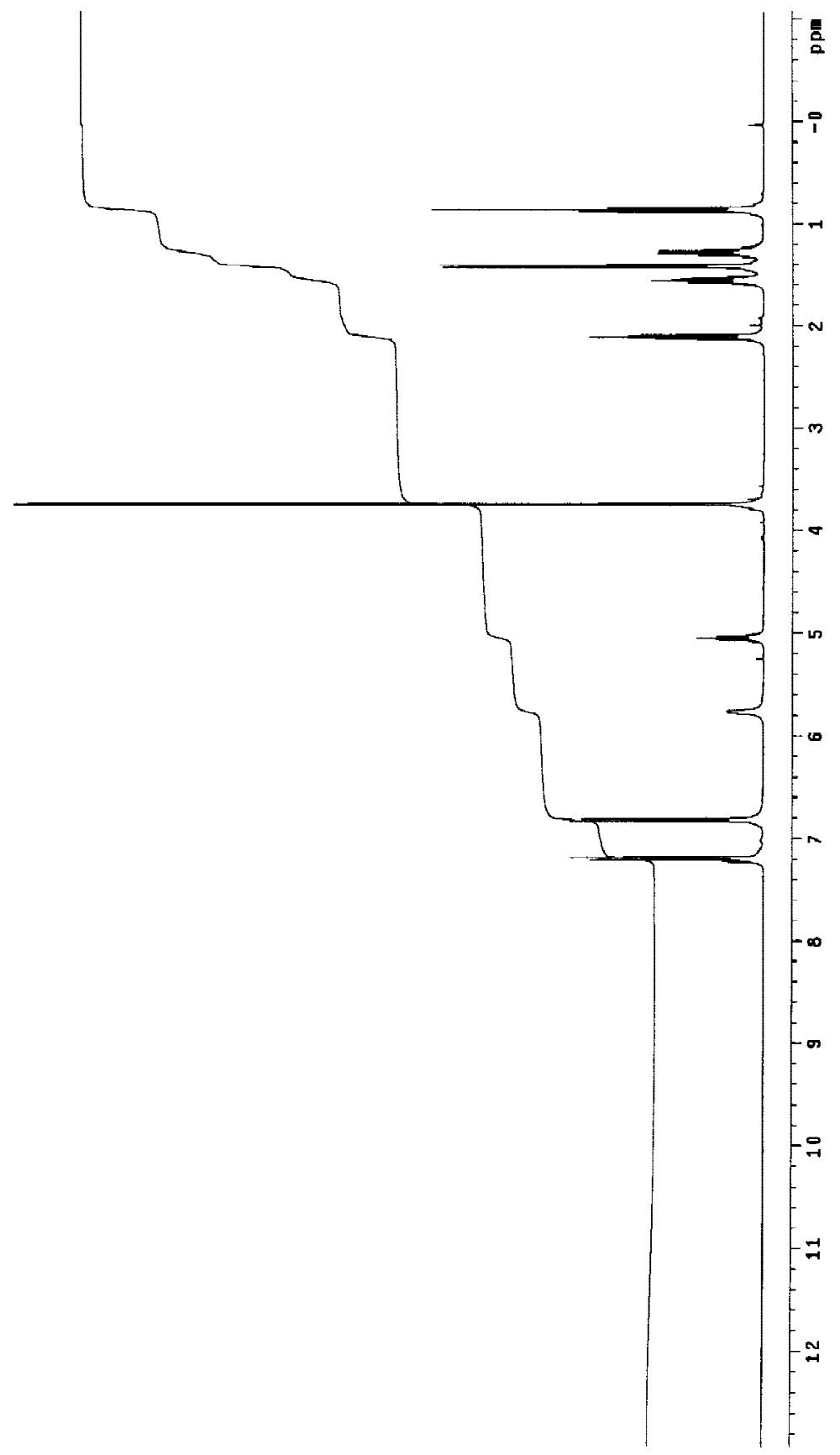


Figure S10. ${ }^{13} \mathrm{C}\left\{{ }^{1} \mathrm{H}\right\}$ NMR spectrum of $[N-1-(p$-methoxyphenyl)ethyl $]$ valeramide.

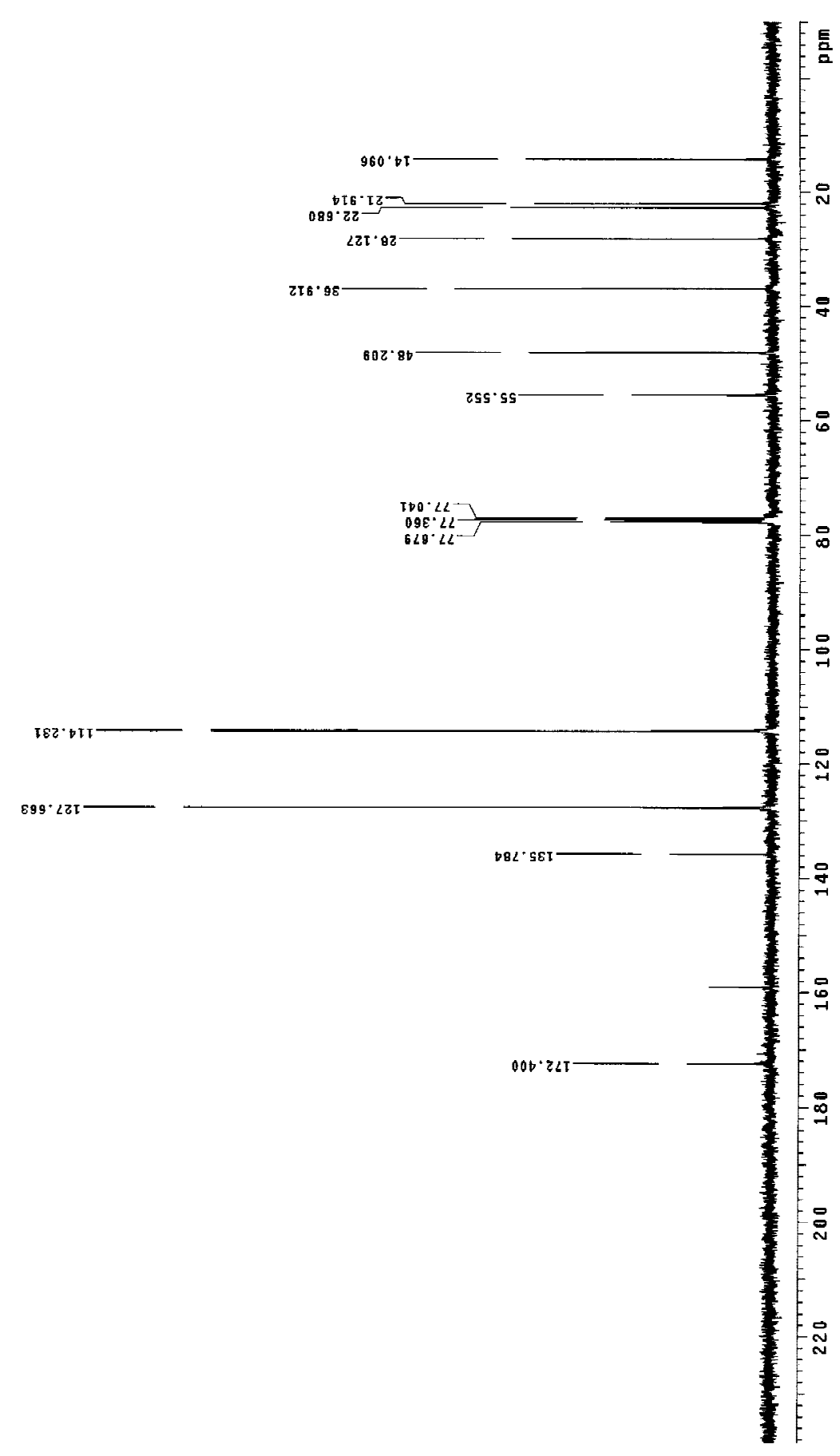




\section{References}

1) Plummer, B. F.; Menendez, M.; Songster, M. J. Org. Chem. 1989, 54, 718.

2) (a) Potapov, V. M.; Dem'yanovich, V. M.; Lazutina, L. I.; Terent'ev, A. P. Zhurnal Obshchei Khimii 1962, 32, 1187. (b) Vaughan, W. R.; Carlson, R. D. J. Am. Chem. Soc. $1962,84,769$.

3) Tsuchimoto, T.; Kamiyama, S.; Negoro, R.; Shirakawa, E.; Kawakami, Y. Chem. Commun. 2003, 852.

4) Noji, M.; Ohno, T.; Fuji, K.; Futaba, N.; Tajima, H.; Ishii, K. J. Org. Chem. 2003, 68, 9340.

5) (a) Smidt, H.; Fischer, A.; Fischer, P.; Schmid, R. D. Biotechnology Techniques 1996, 10, 335. (b) Potapov, V. M.; Dem'yanovich, V. M.; Terent'ev, A. P. Zhurnal Obshchei Khimii 1965, 35, 1340 .

6) Terent'ev, A. P.; Potapov, V. M. Zhurnal Obshchei Khimii 1957, 27, 1092.

7) Moglioni, A. G.; Tombari, D. G.; Iglesias, G. Y. M. J. Chem. Soc., Perkin Trans. 1 1998, 20,3459 .

8) Timokhin, V. I.; Anastasi, N. R.; Stahl, S. S. J. Am. Chem. Soc. 2003, 125, 12996.

9) Au, S.-M.; Huang, J.-S.; Che, C.-M.; Yu, W.-Y. J. Org. Chem. 2000, 65, 7858. 